\title{
Man-machine Integration Design and Analysis System (MIDAS) v5: Augmentations, Motivations, and Directions for Aeronautics Applications
}

\author{
Brian F. Gore, PhD \\ *NASA AMES RESEARCH CENTER \\ Mail Stop 262-4 \\ P.O. Box 1 \\ Moffett Field, CA, USA 94035-0001 \\ brian.f.gore@nasa.gov
}

Keywords: NASA, Human Error, Human Performance Modeling, MIDAS v5, Future System Predictions

\begin{abstract}
As automation and advanced technologies are introduced into transport systems ranging from the Next Generation Air Transportation System termed NextGen, to the advanced surface transportation systems as exemplified by the Intelligent Transportations Systems, to future systems designed for space exploration, there is an increased need to validly predict how the future systems will be vulnerable to error given the demands imposed by the assistive technologies. One formalized approach to study the impact of assistive technologies on the human operator in a safe and non-obtrusive manner is through the use of human performance models (HPMs). HPMs play an integral role when complex human-system designs are proposed, developed, and tested. One HPM tool termed the Man-machine Integration Design and Analysis System (MIDAS) is a NASA Ames Research Center HPM software tool that has been applied to predict human-system performance in various domains since 1986. MIDAS is a dynamic, integrated HPM and simulation environment that facilitates the design, visualization, and computational evaluation of complex man-machine system concepts in simulated operational environments. The paper will discuss a range of aviation specific applications including an approach used to model human error for NASA's Aviation Safety Program, and "what-if" analyses to evaluate flight deck technologies for NextGen operations. This chapter will culminate by raising two challenges for the field of predictive HPMs for complex human-system designs that evaluate assistive technologies: that of (1) model transparency and (2) model validation.

\section{Introduction}

Human Performance Models (HPMs) have traditionally been used to predict sensory processes (Gawron, Laughery, Jorgensen, \& Polito, 1983), aspects of human cognition (Newell, 1990), and human motor responses to system tasks (Fitts \& Posner, 1967; Gore, 2001; Hudlicka \& Corker, 2001). HPM tools are currently undergoing a developmental shift, now being more sensitive to situations that confront a virtual human in systems similar to human-in-the-loop (HITL) situations. HPMs and the human performance modeling process have attempted to integrate operator characteristics (cognitive, attentional, and physical) with environmental characteristics to more accurately represent human-system operations with new, augmented technologies. The growth in HPMs has been to examine human performance in systems including system monitoring (thereby taking information in from the environment) as opposed to the closed-loop view of the human as a mathematical relationship between input and output to a system. These hybrid models that combine closed-loop performance (continuous control), open-loop performance (discrete control) and critical decision-making have been undertaken to represent the "internal models and cognitive function" of the human operator in complex control systems. These hybrid systems involve a critical coupling among humans and machines in a shifting and context sensitive function.
\end{abstract}




\subsection{Using Human Performance Models (HPMs) for Technology Development}

Modeling can play a role in all phases of new technology development from concept development, through the refinement, and deployment process. HPMs provide a flexible and economical way to manipulate aspects of the operator, automation, and task environment to represent the manner that a human engages with the technology under development (Gore, 2002, 2008; Gore, Hooey, Foyle, \& ScottNash, 2008; Hooey \& Foyle, 2008). HPMs have arisen as viable research options due to decreases in computer costs, increases in representative results, and increases in model validity. They are especially valuable because the computational predictions can be generated early in the design phase of a product, system or technology to formulate procedures, training requirements, and to identify system vulnerabilities and where potential human-system errors are likely to arise. The model development process allows the designer to formally examine many aspects of human-system performance with new technologies to explore potential risks brought to system performance by the human operator (Gore \& Smith, 2006). Often this can be accomplished before the notional technology exists for human-in-theloop (HITL) testing (Gore, 2000). More comprehensive results and conclusions can be drawn about technologies being introduced into complex operational environments when used in a cooperative and iterative fashion with HITL simulations. Furthermore, using HPMs in this manner is advantageous because risks to the human operator and costs associated with system experimentation are greatly reduced: no experimenters, no subjects and no testing time (Elkind, Card, Hochberg, \& Huey, 1989; Gore, 2001). HPMs can be used to conduct system robustness testing to evaluate the system from the standpoint of potential deviations from nominal procedures to determine the impact on the performance of the human and the system ("what-if" testing) (Gore \& Smith, 2006; Hooey \& Foyle, 2008).

\section{The Man-machine Integration Design and Analysis System}

MIDAS is a dynamic, integrated human performance modeling and simulation environment that facilitates the design, visualization, and computational evaluation of complex man-machine system concepts in simulated operational environments (Gore, 2008). MIDAS symbolically represents many mechanisms that underlie and cause human behavior (Gore \& Jarvis, 2005). MIDAS combines graphical equipment prototyping, dynamic simulation, and HPMs to reduce design cycle time, support quantitative predictions of human-system effectiveness, and improve the design of crew stations and their associated operating procedures.

\subsection{History}

MIDAS has undergone two paths in its development. The first path termed Air MIDAS focused on specific behaviors in complex human-system interaction and has been applied specifically to aviation operations (Gore, 2002). This development path was entirely code-based with no visualization capability. The second path, termed the NASA MIDAS and currently MIDAS v.5, focused on cross-domain capability (Gore \& Smith, 2006), cognitive behavior model augmentations (Gore, et al., 2008; Gore, Hooey, Wickens, \& Scott-Nash, 2009) and has been applied to a variety of transportation domains Human performance models have produced validated predictions of human performance within complex operating environments ranging from rotorcraft (Atencio, 1994, 1998), nuclear power plan operations (Corker, 1994), space operations (Gore \& Smith, 2006), and commercial aviation (Gore, Hooey, Wickens, \& Scott-Nash, 2009; Hooey, et al., 2010). The NASA MIDAS path contains a comprehensive visualization capability associated with the physical and cognitive operations in their respective contexts. MIDAS links a virtual human, comprised of a physical anthropometric character, to a computational cognitive structure that represents human capabilities and limitations. The cognitive component is comprised of a perceptual mechanism (visual and auditory), memory (short-term, long-term working, and long-term), a decision maker and a response selection architectural component. The complex interplay among bottom-up and top-down processes enables the emergence of unforeseen, and non-programmed behaviors. MIDAS can suggest the nature of pilot errors, and highlight precursor conditions to error such as high levels of memory demand, mounting time pressure and workload, attentional tunneling or 
distraction, and deteriorating situation awareness (SA). MIDAS provides a flexible and economical way to manipulate aspects of the operator, automation, and task environment for simulation analyses.

\subsection{MIDAS v5 Architecture}

Figure 1 illustrates the model's organization and flow of information among the model's components. MIDAS inputs (Figure 1, left column) include the operational environment (e.g., flight profiles, scenario objects and events, etc), the operator tasks and operator process models (e.g., algorithms that represent operator characteristics such as expertise). The MIDAS processing model (Figure 1, middle column) is comprised of a task manager model that schedules tasks to be completed, definitions of the state of models within the physical simulation, a library of "basic" human primitive models that represent behaviors required for all activities such as reach, and cognitive models such as operator perception. The MIDAS output model (Figure 1, right column) generates a runtime display of the task network, the anthropometry as well as mission performance.

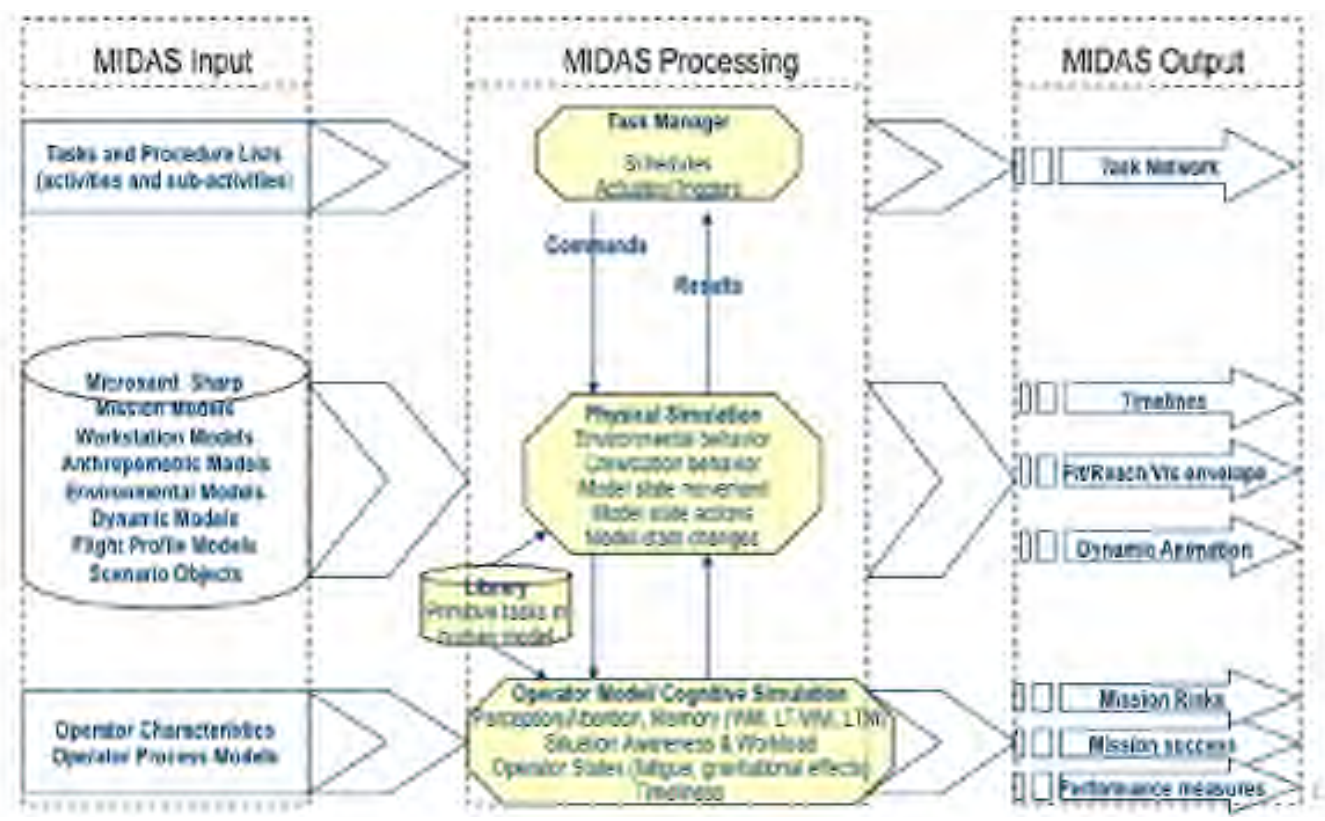

Figure 1. MIDAS Structural Composition and Flow: The agents that make up the MIDAS behavior set are represented as well as the linkage of information passed from one agent to another (adapted from Gore and Smith, 2006).

\subsubsection{MIDAS Input}

Tasks are triggered by information that flows from the environment, through a perception model, to a task network representation of the procedures that then feeds back into the environment. Tasks are characterized by several defining parameters that include conditions under which the task can be performed (e.g., beginning, ending, and wait-for clauses), its relative priority with respect to other tasks, an estimate of its duration for scheduling, its interruption specifications, and the resource required to perform the task defined according to the Modified TAWL (McCracken \& Aldrich, 1984; Mitchell, 2000).

\subsubsection{MIDAS Processing}

\subsubsection{MIDAS Perception}

MIDAS represents perception as a series of stages that information must pass through in order to be processed. The perception model includes visual and auditory information. Visual perception in MIDAS depends on the amount of time the observer dwells on an object and the perceptibility of the observed 
object. The perception model computes the perceptibility of each object that falls into the operator's field of view based on properties of the observed object, the visual angle of the object and environmental factors. In the current implementation of MIDAS, perception is a three-stage, time-based perception model (undetected, detected, comprehended) for objects inside the workstation (e.g., an aircraft cockpit) and a four-stage, time-based perception model (undetected, detected, recognized, identified) for objects outside the workstation (e.g., taxiway signs on an airport surface). The model computes the upper level of detection (i.e., undetectable, detectable, recognizable, identifiable for external objects) that can be achieved by the average unaided eye if the observer dwells on it for a requisite amount of time. For example, in a low-visibility environment, the presence of an aircraft on the airport surface may be 'detectable' but the aircraft company logo on the tail might not be 'recognizable' or 'identifiable' even if he/she dwells on it for a long time.

\subsubsection{MIDAS Memory}

Tasks from the MIDAS input process also require knowledge held either in the operator's memory (working, long-term working, and long-term) or available from the environment to be consulted and used to determine subsequent tasks to be completed (Gore, Hooey, Wickens, \& Scott-Nash, 2009). Memory is represented as a three stage, time decay model ${ }^{1}$. The stages are working memory (WM), long-term working memory (LT-WM), and long-term memory (LTM). The decay rates cause memory to be above or below a "retrievability" threshold based on the time since the information was last accessed. The retrievability thresholds incorporated into MIDAS are 5 seconds for WM and 5 minutes (300 seconds) for LT-WM. The WM decay rate is faster than the LT-WM decay rate. Information that falls below the retrevability threshold is forgotten. This causes the perception level to be set to Undetected for external visual and auditory information or Unread for internal visual information. Newly perceived and recently refreshed attributes will be retained in LT-WM only if a node with newly updated or referenced attributes leaves WM before its attributes have decayed below the retrievability threshold. An operator may retain newly perceived information after it leaves WM, at least for a while, until it decays below the LT-WM retrievability threshold. If the information necessary for activity performance is available, and its priority is sufficient to warrant performance, then the schedule within the model operates according to heuristics that can be selected by the analyst. In most cases the heuristic is to perform activities concurrently when that is possible, based on knowledge and resource constraints.

\subsubsection{MIDAS Visual Attention}

MIDAS' attention-guiding model operates according to the SEEV model (Gore, Hooey, Wickens, \& Scott-Nash, 2009). SEEV estimates the probability of attending, P(A), to an area of interest in visual space, as a linear weighted combination of the four components - salience, effort, expectancy, and value. Attention in dynamic environments is driven by the bottom-up capture of Salient $(S$ ) events (e.g., a flashing warning on the instrument panel) and inhibited by the Effort (E) required to move attention (e.g., a pilot will be less likely to scan an instrument located at an overhead panel, head down, or to the side where head rotation is required, than to an instrument located directly ahead on a head-up display (HUD) (Wickens, Goh, Helleberg, Horrey, \& Talleur, 2003). The SEEV model also predicts that attention is driven by the Expectancy (EX) of seeing a Valuable (V) event at certain locations in the environment. $\mathrm{SEEV}$ is driven per the following equation ${ }^{2}$ :

$$
\mathrm{P}(\mathrm{A})=\mathrm{s} * \mathrm{~S}-\mathrm{ef} * \mathrm{EF}+\mathrm{ex}^{*} \mathrm{EX}+\mathrm{v} * \mathrm{~V}
$$

\footnotetext{
${ }^{1}$ In contrast to MIDAS v5, memory in Air MIDAS is represented as a two-stage model of WM and LTM (Baddeley \& Hitch, 1974).

${ }^{2}$ Properties of a display or environment are illustrated by coefficients in uppercase characters, while those in lower case describe the weight assigned to those properties in the control of an operator's attention (Wickens, et al., 2008).
} 
The four SEEV parameters drive the visual attention around an environment such as the dynamic cockpit in a computational version of this model. For example, the simulated eyes following the model will fixate more frequently on areas with a high bandwidth (and hence a high expectancy for change), as well as areas that support high-value tasks, like maintaining stable flight (Wickens, Hooey, Gore, Sebok, \& Koenecke, 2010). SEEV has been under development since 2001 and has been extensively validated with empirical human-in-the-loop data from different domains (Gore, Hooey, Wickens, Sebok, et al., 2009; Wickens \& McCarley, 2008).

The integration of the SEEV model into MIDAS v5 allows dynamic scanning behaviors by calculating the probability that the operator's eye will move to a particular AOI given the tasks the operator is engaged in within the multitask context. It also better addresses allocation of attention in dynamic environments such as flight and driving tasks.

\subsubsection{MIDAS Output}

The MIDAS outputs include the task network model, anthropometric model and Computer Aided Design (CAD) visualizations (using the jack $^{\mathrm{TM}^{\mathrm{Tm}}}$ software), timelines of workload and SA, and risk vulnerabilities as inferred from timeline violation of optimal response times, workload spikes, or SA violations. The complex interplay among bottom-up and top-down processes enables the emergence of unforeseen, and non-programmed behaviors (Gore \& Smith, 2006). MIDAS can suggest the nature of operator errors, and highlight precursor conditions to error such as high levels of memory demand, mounting time pressure and workload, attentional tunneling or distraction, and deteriorating SA. An illustration of the integration of the different models in a recent aeronautics simulation completed with MIDAS v5 can be found in Figure 2.

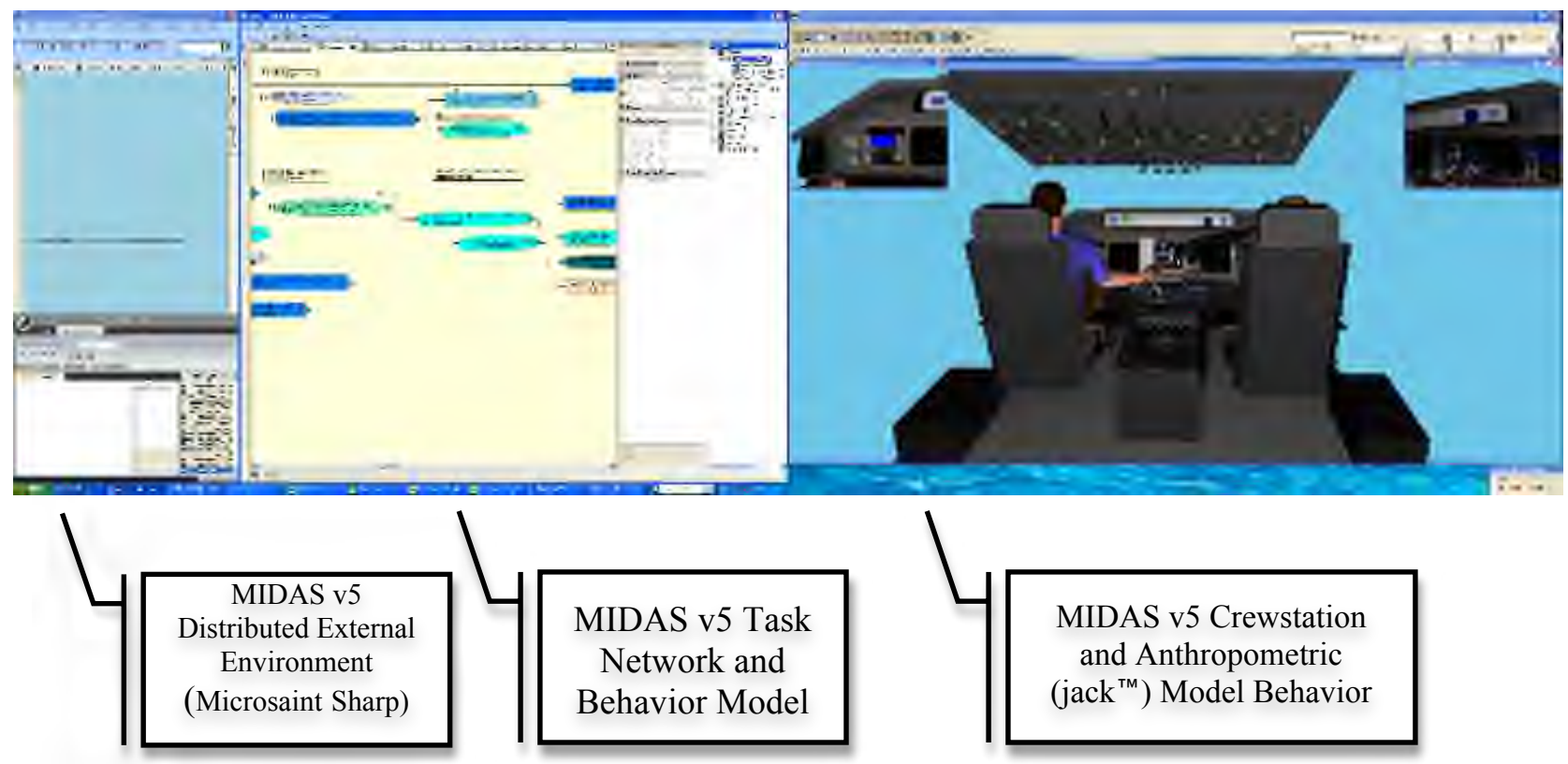

Figure 2. MIDAS' Environment, Task, and Anthropometric Models.

\footnotetext{
${ }^{\mathrm{rm}}$ jack" is maintained by Siemens PLM Solutions.
} 


\section{Human Performance Modeling of Next Generation Air Transportation Systems}

The current air traffic control (ATC) system in the United States will not be able to manage the predicted two to three times growth in air traffic (JPDO, 2009). The Next Generation Air Transportation System (NextGen) is a future aviation concept that has as its goals to increase the capacity, safety, efficiency, and security of air transportation operations (JPDO, 2009). Two MIDAS HPMs will be highlighted. The first illustrates a human error model of an aviation surface-related application that uses some candidate NextGen concepts as generated by Air MIDAS, and the second illustrates a recent application of NASA's MIDAS in the context of NextGen approach and land operations.

\subsection{Human Error Modeling}

New conceptual designs especially those being developed for complex systems are likely to incorporate technologies that utilize or rely on a human's cognitive capabilities. New conceptual designs often incorporate automation to assist the human operator in their task performance. Automation increases precision and economy of operations but can have the unanticipated effect of increasing a human operator's cognitive, perceptual and attentional workload (Sarter, Woods, \& Billings, 1997). The increase in workload often negates some of the benefits afforded to the system from the use of automation. Operators may miss critical events in the environment due to a number of unexpected human-automated systems issues such as unevenly distributed workload, new attentional demands, and new coordination demands among operators (Sarter, et al., 1997). When critical physical events are missed, no response is possible and human errors occur (Hollnagel, 1993, 2000).

Quantifying human error in a manual control representation is a task that can be modeled quite simply by control input-output mechanisms exemplified by the servo-mechanisms developed in the mid-1940's (Craik, 1947). The identification of the underlying reasons for the error exhibited by human performance, however is much more difficult. The reasons for inaccurate human performance could be the result of inadequate design of the physical work environment or could be due to the human cognitive component. Many theories exist on human error causality and all have some merit as they attempt to quantify the measurement of human error (Hollnagel, 1993; Hollnagel \& Woods, 2005; Norman, 1981; Rasmussen, 1976; Reason, 1990).

\subsubsection{Modeling Human Error}

An integrated flight deck model of ramp navigation and gate-approach at the Chicago O'Hare Airport (ORD) was used to produce human error performance when technological introductions that took the form of augmented flight deck concept displays (T-NASA; Foyle, Andre, \& Hooey, 2005) were made to current day operations using the Air MIDAS HPM (Gore, Verma, Jadhav, Delnegro, \& Corker, 2002 ). In order to generate a sufficiently valid model of error predictions, the equipment (physical aircraft), the crewstation and the external environment were modeled at varying levels of fidelity depending on the importance of the information for updating the operator's world. The control modes in Air MIDAS that had the potential of being sensitive to manipulations include memory errors and their effect on the simulated crew's internal representation, termed updateable world representation (UWR). Three types of error mechanisms were represented.

- The first error type, declarative memory errors, included errors that occurred when virtual operators forgot the active procedure as a result of having too many procedures of the same type operating at the same time. The occurrence of this error was modeled by scheduling the simulation to cause multiple competing behaviors to occur concurrently and invoke the procedure scheduler (dropped tasks $=$ memory loss).

- The second error type, memory load errors, occurred as a result of information competing for WM space. When there were a number of items needing to occupy WM, one item in WM needed to be 
shifted out of the limited capacity store by the subsequent information from the pilot or from the controller communication. This information was lost if it not written down to a location from an actively available list from which the operator was able to visually encode the information (for example, a taxi clearance).

- The third type of error, UWR discrepancy errors, occurred when there was a worldview inconsistency between two virtual operators. This error occurred when one virtual operator erroneously "thought" a different virtual operator had received information. This UWR discrepancy was a possible cause of and a response to the occurrence of an error.

The errors represented above were created by increasing the number of items in WM and by increasing information transfers through active procedures. Each type of error emerged as a result of the scenario requirements and demands placed on the virtual operators and each type of error was not created deterministically.

The Air MIDAS software operated with different human operator model memory decay rates of 120 seconds and 40 seconds. Environment triggers (e.g., turns, signs, ATC calls) elicited the human performance. Error rates as measured by missing turns, operator performance times and workload were output from the HPM. Each of these individual elements was considered to be critical in the analysis of error for determining the conditions under which the error rate changes in response to operational changes. In the Air MIDAS model, errors occurred as a result of increasing decay rates and reducing memory capacity. As this effort predicts, the model loading factors appear to have an impact on the performance of the forgetting mechanism and on invoking the rule guiding the response to the forgetting function. This demonstrated that the computational mechanisms within the Air MIDAS software are attempting to replicate the operations of humans when humans are faced with a forgotten piece of information. When there are a number of items occupying WM, one item in WM is shifted out of the limited capacity store by the subsequent information from the pilot or from the controller communication. Each type of error was therefore not created deterministically; rather each error type emerged based on the environmental requirements and loads that were associated with the performance requirements of the agent. A prediction for increased auditory and cognitive demands as time in the scenario increased (as the virtual operator approached the second turn) was also found. The Air MIDAS model provided useful information for identifying risk factors that increase the probability of error and was useful for providing some insight into mitigation strategies when errors occur. Air MIDAS did not however predict error rates.

\subsection{2 "What-If" Next Gen Approach and Landing Application}

MIDAS v5 has been applied to examine a NextGen approach to land concept termed the very closely spaced parallel approach/operations (VCSPA/O). Based on Raytheon's Terminal Area Capacity Enhancement (TACEC) parallel approach procedures, VCSPA/O requires that runway spacing be reduced (Verma, Lozito, \& Trott, 2008). This reduction in distance between the runways increases the likelihood of wake vortex incursion during independent simultaneous operations. VCSPA/O requires that a safe and proper breakout maneuver be calculated and presented via new displays to the cockpit crew (Verma, et al., 2008). In order to evaluate the VCSPA/O concept, two MIDAS v5 models were generated. The first was a Simultaneous Offset Instrument Approach (SOIA) model that contained the current-day procedures, and the second was a NextGen VCSPA/O model that contained advance displays of traffic and wake information in the cockpit and a modification to the roles and responsibilities of the flight crew and ATC modeled operators. The advanced technology in the "NextGen" VCSPA/O condition enabled closer separation in low visibility, lower landing minima, autoland capability, and enhanced wake and traffic data. This MIDAS model involved over 500 tasks and culminated in a verifiable model of approach and land operations (vetted during the model building process by Subject Matter Experts; SMEs). One hundred Monte Carlo model runs were generated by MIDAS. Performance profiles along the variables of operator workload, visual attention, and cockpit alert detection times for both the captain and 
first officer during the descent, approach, and land phases of flight were collected. Both conditions were run in IMC with no out the window (OTW) visibility. The SOIA flight crew broke out of the clouds at 2100' and maintained separation from traffic and monitored runway alignment OTW. The VCSPA modeled flight crew monitored traffic separation and wake information on the ND throughout the approach and broke out of the clouds at 100'. This model effort illustrated the "what-if" simulation capability within MIDAS. The "what-if" approach was completed when MIDAS was exercised with one set of displays and procedure sets designed to represent current day operations and roles followed by a second simulation with an alternate set of displays and procedures encoded to represent the NextGen displays and expected procedures.

Important insights regarding the impact of NextGen VCSPA/O operations on pilot workload, visual attention, and alert detection times were revealed through this research. The MIDAS model predicted increased workload during descent and initial approach due to increased information available (traffic, weather, wake) on the flight deck but reduced workload during final approach and land due to automated landing procedures and ease of information retrieval (traffic and runway alignment) in the NextGen condition. Technologies that shift the workload demands away from the visual modality using auditory and haptic displays should be pursued as NextGen operations may tax the visual and cognitive-spatial channels to a greater extent than current day operations during specific phases of flight. Furthermore, the NextGen condition suggested a more balanced workload across the descent, approach, and land phases of fligh than current day operations. In terms of visual attention, NextGen condition may draw visual attention to the navigation display (ND), which suggest that the pilots will more likely be heads-down during the critical minutes before touchdown (TD). Increased head-down time within the cockpit due to the presence of additional, more salient, information, may draw pilots' attention into the cockpit at inopportune times leaving pilots vulnerable to external hazards (other aircraft or obstacles on the runway, terrain). It is important to remember that other instantiations of the VCSPA/O concept (with different operational requirements) may reveal different human-system vulnerabilities.

This MIDAS v5 effort led to a greater awareness of potential parameters such as the change in roles and responsibilities in the NextGen that should be included in system designs and enabled the research program to visualize the interactions that are likely in future NextGen operations. It is anticipated that additional validation approaches will be developed and applied to the VCSPA/O require an increased number of alternative closely spaced operations for additional "what-if" scenarios including alternative pilot roles and responsibilities, and information requirements.

\section{Conclusion}

Automation often changes the nature of the human's role in the system. Therefore, as automation and technologies are developed, it becomes increasingly important to predict how the human operator perceives and responds to the automation. MIDAS has proven useful for identifying general humansystem vulnerabilities and cross-domain error classes and for recommending mitigation strategies and system re-designs to account for the vulnerable areas, or risks, in system design (Gore \& Smith, 2006). Fundamental design issues can therefore be identified early in the design lifecycle, often before hardware simulators and HITL experiments can be conducted. HPMs are most useful when used cooperatively with HITL simulations to supplement the HITL research. HPMs like MIDAS provide an easy to use and cost effective means to conduct experiments that explore "what-if" questions about concepts of operation (CONOPS) in specific domains of interest.

A number of significant challenges exist for the state of the art in HPMs, two of which will now be highlighted.

\subsection{Transparency}

The first challenge relates to model transparency. Model transparency refers to the ability to comprehend the relationships that exist among the models being used in the simulation, the performance of the models 
in the simulation, which models are triggering in the model architecture, and whether the model is behaving as the model developer would expect (Gore, 2008). Other researchers refer to this as model traceability, model behavior visibility, model verifiability, and model interpretability (Elkind, et al., 1989; Gluck \& Pew, 2005; Hooey \& Foyle, 2008; Napierski, Young, \& Harper, 2004). Transparency in integrated HPMs is needed to support model verification, validation, and credibility. However, model transparency can be difficult to attain because of the complex interactions that can exist among the cognitive, physical, environment and crew station models, and because the cognitive models embedded within integrated HPMs produce behaviors that are not directly observable. Three types of transparency that the MIDAS researchers have found useful to understand, interpret, and increase the confidence in the complex models' output include transparency of the input, transparency of the integrated architecture, and transparency of the output (Gore, et al., 2008).

\subsection{Validation}

The second challenge facing the HPM community is validation. Validation remains a very large challenge for the HPMs community because statistical validation is oftentimes seen as the Holy Grail for determining whether a model is suitable but when models are deemed statistically valid, they generalize less, and are less re-usable for applications in new contexts. This places the field of modeling into the conundrum of making models that are statistically valid (i.e. a high correlation between predicted and actual data) but that lack the ability to generalize to other tasks or scenarios. When the generalizability of the model is limited, then its value as a cost-effective approach to predict complex human-system interactions is reduced.

Validation is further challenged when modeling future technology concepts where no or little HITL data exists upon which to statistically validate a model (as in the NextGen aviation systems or concepts being designed for the Space program). It is argued that the definition of model validation must be expanded beyond that of statistical results validation to be more representative of a 'model develop - model verify model manipulate - model validate' iterative process, a process that is currently underway in MIDAS' FAA modeling of NextGen operations. The model develop phase of an HPM effort is one that is comprised primarily of model verification, where the inputs parameters such as the SEEV weights and workload primitives, are built from and operate as expected given the model's context. Model verification is the process of determining whether a simulation model and its associated data behave as intended by the model developer / analyst (Sargent, 1980). The model manipulate phase is where the model's conceptual parameters are manipulated to bring the overall model performance closer to expectations. The model validation phase involves determining the degree to which a model or simulation and its associated predictions are an accurate representation of the real world, from the perspective of the intended users of the model or simulation (Balci, 1988; Law \& Kelton, 2000; Sargent, 1980). Formalized 'developvalidate' iteration cycles are an important step toward increasing the credibility of HPMs particularly as the complexity of human-system operations increases.

\section{References}

Atencio, A. (1994). Air Warrior MIDAS. Retrieved from http://caffeine.arc.nasa.gov/midas/originalmidas/Air Warrior.html

Atencio, A. (1998). Short Haul Civil Tiltrotor MIDAS. Retrieved from http://caffeine.arc.nasa.gov/midas/originalmidas/Tiltrotor_MIDAS.html.

Baddeley, A.D., \& Hitch, G. (1974). Working memory. In G.H. Bower (Ed.), The psychology of learning and motivation. London: Academic Press.

Balci, O. (1988). Verification, validation, and testing techniques. In Jerry Banks (Ed.), Handbook of simulation: Principles, methodology, advances, applications, and practice (pp. 335-427): N.Y.: Wiley \& Sons, Inc.

Corker, K. (1994). Man-machine integration design and analysis system (MIDAS) applied to a computer-based procedure-aiding system. Paper presented at the 38th Annual Meeting of the Human Factors and Ergonomics Society. 
Craik, K.J.W. (1947). Theory of the human operator in control systems. I. The operator as an engineering system. British Journal of Psychology, 38, 56-61.

Elkind, J., Card, S.K., Hochberg, J., \& Huey, B.M. (1989). Human performance models for computer-aided engineering. Washington, D.C.: National Academy Press.

Fitts, P.M., \& Posner, M.A. (1967). Human Performance. Pacific Pallisades, CA: Brooks/Cole.

Foyle, D.C., Andre, A.D., \& Hooey, B.L. (2005). Situation awareness in an augmented reality cockpit: Design, viewpoints and cognitive glue. Paper presented at the 11th International Conference on Human Computer Interaction, Las Vegas, NV.

Gawron, V.J., Laughery, K.R., Jorgensen, C.C., \& Polito, J. (1983, May). A Computer Simulation Of Visual Detection Performance Derived From Published Data. Paper presented at the Proceedings of the Ohio State University Aviation Psychology Seminar, Columbus, Ohio.

Gluck , K.A., \& Pew, R.W. (2005). Modeling human behavior with integrated cognitive architectures: comparison, evaluation and validation. In K.A. Gluck \& R.W. Pew (Eds.), Modeling human behavior with integrated cognitive architectures: comparison, evaluation and validation. New Jersey: Lawrence Erlbaum \& Associates.

Gore, B.F. (2001). The study of distributed cognitiion in free flight: A human performance modeling tool structural comparison. SAE Transactions - Journal of Passenger Cars: Mechanical Systems, 109(6), 2291-2296.

Gore, B.F. (2002). Human performance cognitive-behavioral modeling: A benefit for occupational safety. International Journal of Occupational Safety and Ergonomics (JOSE), 8(3), 339-351.

Gore, B.F. (2008). Chapter 32: Human performance: Evaluating the cognitive aspects. In V. Duffy (Ed.), Handbook of digital human modeling (pp. 32-31-32-18). Boca Raton, Fla: CRC Press Inc.

Gore, B.F., Hooey, B.L., Foyle, D.C., \& Scott-Nash, S. (2008, July 14-17). Meeting the challenge of cognitive human performance model interpretability though transparency: MIDAS v5.x. Paper presented at the Applied Human Factors and Ergonomics International Conference, Las Vegas, Nevada.

Gore, B.F., Hooey, B.L., Wickens, C.D., \& Scott-Nash, S. (2009, July19-24). A computational implementation of a human attention guiding mechanism in MIDAS v5. Paper presented at the 12th International Conference, HCI International 2009, San Diego, CA.

Gore, B.F., Hooey, B.L., Wickens, C.D., Sebok, A., Hutchins, S, Salud, E., et al. (2009). Identification of NextGen Air Traffic Control and Pilot Performance Parameters for Human Performance Model Development in the Transitional Airspace (No. NASA Final Report: NRA \#NNX08AE87A). San Jose, CA: San Jose State University.

Gore, B.F., \& Jarvis, P. (2005). New integrated modeling capabilities: MIDAS' recent behavioral enhancements. SAE Transactions - Journal of Passenger Cars: Electronic and Electrical Systems, 752-760.

Gore, B.F., \& Smith, J.D. (2006). Risk assessment and human performance modeling: the need for an integrated approach. International Journal of Human Factors of Modeling and Simulation, 1(1), 119-139.

Gore, B.F., Verma, S., Jadhav, A., Delnegro, R., \& Corker, K.M. (2002). Human error modeling predictions: Air MIDAS human performance modeling of T-NASA. San Jose, CA: San Jose State University.

Hollnagel, E. (1993). Human reliability analysis: Context and control. In B.R. Gaines \& Monk A. (Ed.), Computers and people series. New York: Academic Press.

Hollnagel, E. (2000). Modeling the orderliness of human action. In N.B. Sarter \& R. Amalberti (Eds.), Cognitive engineering in the aviation domain (pp. 65-98). New Jersey: Lawrence Erlbaum Associates.

Hollnagel, E., \& Woods, D. D. (2005). Joint cognitive systems: Foundations of cognitive systems engineering. NY: Taylor \& Francis.

Hooey, B.L., \& Foyle, D.C. (2008). Advancing the state of the art of human performance models to improve aviation safety. In B.L. Hooey \& D.C. Foyle (Eds.), Human performance modeling in aviation (pp. 321349). Boca Raton: CRC Press/Taylor \& Francis.

Hooey, B.L., Gore, B.F., Wickens, C.D., Salud, E., Scott-Nash, S., Socash, C., et al. (2010, June 30-July 2). Modeling Pilot Situation Awareness. Paper presented at the Human Modelling of Assistive Technologies Workshop, Begirate, Italy.

Hudlicka, E., \& Corker, K. (2001). Affective Context and Control in Human-System Modeling. Paper presented at the Human Error, Safety and System Design International Workshop.

JPDO, Joint Planning and Development Office (2009). Concept of operations for the next generation air transportation system. Joint Planning and Development Office (Vol. 3). Washington, DC: JPDO.

Law, A.M., \& Kelton, D.W. (2000). Simulation, modeling and analysis (3 ed.). NY: McGraw Hill.

McCracken, J.H., \& Aldrich, T.B. (1984). Analysis of selected LHX mission functions: Implications for operator workload and system automation goals. Fort Rucker, AL: Anacapa Sciences, Inc. 
Mitchell, D. K. (2000). Mental workload and ARL workload modeling tools. Aberdeen Proving Ground, MD: U.S. Army Research Laboratory.

Napierski, D., Young, A., \& Harper, K. (2004, May). Towards a common ontology for improved traceability of human behavior models. Paper presented at the Proceedings of the 13th Conference on Behavior Representation in Modeling and Simulation, Arlington, VA.

Newell, A. (1990). Unified Theory of Cognition. Cambridge: Harvard University Press.

Norman, D. (1981). Categorization of action slips. Psychological Review, 88, 1-15.

Rasmussen, J. (1976). Outlines of a hybrid model of the process operator. In T.B. Sheridan \& G. Johanssen (Eds.), Monitoring Behavior and Supervisory Control (pp. 371-384). New York: Plenum Press.

Reason, J.T. (1990). Human Error. Cambridge: Cambridge University Press.

Sargent, R.G. (1980). Validation of simulation models. Paper presented at the 1st Winter Simulation Conference.

Sarter, N.B., Woods, D.D., \& Billings, C.E. (1997). Automation surprises. In G. Salvendy (Ed.), Handbook of Human Factors and Ergonomics (pp. 1926-1943). NY: New York: Wiley, Inc.

Verma, S., Lozito, S., \& Trott, G. (2008). Preliminary Guidelines on Flight Deck Procedures for Very Closely Spaced Parallel Approaches. Paper presented at the International Council for the Aeronautical Sciences (ICAS) 2008 Congress.

Wickens, C.D., Goh, J., Helleberg, J., Horrey, W., \& Talleur, D.A. (2003). Attentional Models of Multi-Task Pilot Performance using Advanced Display Technology. Human Factors, 45(3), 360-380.

Wickens, C.D., Hooey, B.L., Gore, B.F., Sebok, A., \& Koenecke, C. (2010). Identifying Black Swans in NextGen: Predicting Human Performance in Off-Nominal Conditions. Human Factors, 51(5), 638-651.

Wickens, C.D., \& McCarley, J.S. (2008). Applied Attention Theory. Boca Raton, Fla: CRC Press, Taylor and Francis Group.

Wickens, C.D., McCarley, J.S., Alexander, A., Thomas, L., Ambinder, M., \& Zheng, S. (2008). Attention-Situation Awareness (A-SA) model of pilot error. In D.C. Foyle \& B.L. Hooey (Eds.), Human Performance Modeling in Aviation. Boca Raton, Fla: CRC Press, Taylor and Francis.

\section{Author Biography}

Dr. BRIAN GORE is a Principal Investigator for San Jose State University Research Foundation, and is NASA's Technical Lead for the Man-machine Integration Design and Analysis System (MIDAS) at the NASA Ames Research Center in California. Dr. Gore manages the MIDAS applications and provides the direction for MIDAS' development. Dr. Gore's Human Factors and Ergonomics (HFE) research experience spans system design in surface transportation and aerospace domains using human in the loop and computational modeling simulation methodologies. The last 14 years of Dr. Gore's HFE experience has been at NASA Ames in the Human Systems Integration Research Division where he has been leading efforts aimed at augmenting human performance model capabilities with MIDAS.

\section{Acknowledgment}

The composition of this work was supported by the Federal Aviation Authority (FAA)/NASA Inter Agency Agreement DTFAWA-10-X-80005 Annex 5. The author would like to thank all reviewers for their insightful comments. 